\title{
Quantification of Disturbed Coronary Blood Flow: Disturbed Vorticity Index and its Relation With Fractional Flow Reserve
}

Miao Chu, BSc${ }^{1,2}$, Clemens von Birgelen, $\mathrm{MD}, \mathrm{PhD}^{3}$, Yingguang Li, $\mathrm{MSc}^{4}$, Jelmer Westra, MS ${ }^{5}$, Junqing Yang, MD $^{6}$, Niels R. Holm, MD ${ }^{5}$, Johan H.C. Reiber, $\mathrm{PhD}^{4}$, William Wijns, $\mathrm{MD}, \mathrm{PhD}^{7}$, and Shengxian $\mathrm{Tu}, \mathrm{PhD}^{1,2,4}$

1. Biomedical Instrument Institute, School of Biomedical Engineering, Shanghai Jiao Tong University, Shanghai, China

2. Shanghai Med-X Engineering Research Center, Shanghai Jiao Tong University, Shanghai, China

3. Department of Cardiology, Thoraxcentrum Twente, MST, Enschede, the Netherlands

4. Division of Image Processing, Leiden University Medical Center, the Netherlands

5. Department of Cardiology, Aarhus University Hospital, Skejby, Denmark

6. Department of Cardiology, Guangdong General Hospital, Guangzhou, China

7. The Lambe Institute for Translational Medicine and Curam, National University of Ireland, Galway, and Saolta University Healthcare Group, Galway, Ireland

Brief title: Disturbed Coronary Blood Flow and FFR

Word count: abstract: 244; manuscript (from introduction; including references and figure legends): 4,752

Tables/figures: $1 / 6$

Web-based videos: 1

References: 30

Funding: The study was supported by the Natural Science Foundation of China (Grant No. 31500797 and 81570456) and the National Key Research and Development Program of China (Grant No. 2016YFC0100500).

Conflicts of interest: $\mathrm{C}$ von Birgelen has been unpaid consultant to device-manufacturing companies; his institution has received research grants, provided by AstraZeneca, Biotronik, Boston Scientific, and Medtronic. YL is an employee of Medis with a research appointment at the Leiden University Medical Center. NR Holm NRH has received research grants from Abbott, Terumo, Medis medical imaging, and Boston Scientific. JHC Reiber is the CEO of Medis, and has a part-time appointment at Leiden University Medical Center as Prof of Medical Imaging. Cardiovascular Research Center Aalst (previous affiliation of W Wijns) has 
received institutional grant support and consultancy fees on his behalf. S Tu and J Westra received research grants from Medis medical imaging systems bv. All other authors declare that they have no conflict of interest.

\section{Acknowledgements:}

S Tu acknowledges the support by The Program for Professor of Special Appointment (Eastern Scholar) at Shanghai Institutions of Higher Learning (No. TP2015014).

\section{Address of correspondence:}

Prof. Shengxian Tu, PhD, FESC, Room 123, Med-X Research Institute, Shanghai Jiao Tong University, No. 1954, Huashan Road, Shanghai 200030, PR China.

Phone: +8621 62932631 - Fax: +862162932156 - E-mail: sxtu@sjtu.edu.cn 


\begin{abstract}
Objective To develop a novel hemodynamic index to quantify disturbed coronary blood flow and investigate its relationship with lesion-associated pressure drop, and fractional flow reserve (FFR).

Background The relation between FFR and local coronary flow patterns is incompletely understood.

Methods Three-dimensional angiographic reconstruction and computational fluid dynamics were applied to simulate a patient's pulsatile coronary flow. A novel parameter, disturbed vorticity index (DVI), was derived to quantify the stenosis-induced flow disturbance. The relation between DVI and pressure drop was assessed in 9 virtual vessel models with different obstruction characteristcs. In addition, we evaluated the correlation between DVI, FFR, hyperemic flow velocity, and anatomic parameters, using data of 84 intermediate lesions from 73 participants in an international, multicenter FFR-study.
\end{abstract}

Results In our obstructed virtual vessel models, heterogeneous flow vorticity accompanied disturbed flow patterns. DVI increased with increasing flow rate, stenosis severity, and lesion complexity. There was an excellent correlation between DVI and pressure drop across all models with a determination coefficient $\mathrm{R}^{2}=0.85 \quad(\mathrm{p}<0.001)$. In-vivo, DVI showed a correlation with FFR $(\rho=-0.74, p<0.001)$ that was stronger than the relations of FFR with hyperemic flow velocity $(\rho=-0.27, p=0.015)$, lesion length $(\rho=-0.36, p=0.001)$ and percent diameter stenosis $(\rho=-0.40$, $\mathrm{p}<0.001)$.

Conclusions DVI, a novel index to quantify disturbed flow, was related to pressure drop in virtual obstructed vessel models and showed a strong inverse relation with FFR in intermediate human coronary lesions. It supports the prognostic value of FFR and might be used to improve image-based computation of FFR. 


\section{Condensed Abstract}

We developed a novel index to quantify disturbed coronary flow and investigated its relation with stenosis-induced pressure drop in virtual vessel models and with FFR in-vivo. In models, the disturbed vorticity index (DVI) increased with increasing flow rate, and with stenosis severity and complexity. Furthermore, DVI correlated with trans-lesional pressure drop across all models. In 84 intermediate coronary lesions, DVI showed with FFR a strong inverse relation that was stronger than the relations of FFR with hyperemic flow velocity, lesion length, and percent diameter stenosis. DVI supports the prognostic value of FFR and may improve the image-based computation of FFR.

\section{Key Words}

Vascular hemodynamics

Flow patterns

Coronary angiography

Coronary artery disease

Fractional flow reserve
Abbreviations
$3 \mathrm{D}=$ three-dimensional
$\mathrm{CFD}=$ computational fluid dynamics
$\mathrm{DS} \%=$ percent diameter stenosis
DVI $=$ disturbed vorticity index
$\mathrm{FFR}=$ fractional flow reserve
$\mathrm{LL}=$ lesion length 


\section{Introduction}

Various clinical studies have demonstrated that guidance of percutaneous coronary interventions based on pressure wire-derived fractional flow reserve (FFR) improves the outcome of patients, treated for symptomatic coronary artery disease, and lowers costs(1-4). Despite the evidence in favor of FFR as a clinically relevant tool, the association of the 'gross index' FFR with local coronary flow patterns is not fully understood. It is generally believed that locally disturbed flow plays an important role in the formation and progression of atherosclerotic lesions(5-7), plaque destabilization(8,9) and vascular response to coronary stenting(10). Besides that, a significant disturbance of laminar flow may contribute to FFR beyond the established factors, such as lumen stenosis and lesion length(11).

Vorticity, an important flow parameter to characterize locally disturbed flow, describes the tendency of fluid to rotate(12). The magnitude of vorticity inherently depicts both the flow rate and its directional information. Previous studies have demonstrated that vorticity is relevant to turbulent flow as a result of lumen obstruction, local vessel expansion, or vessel motion; however, in these studies vorticity was assessed qualitatively rather than quantitatively(13-15).

In the present study, we developed a novel measure to quantify locally disturbed coronary flow, hereafter named 'disturbed vorticity index' (DVI). We then performed computer-based (virtual) experiments to investigate the relation between DVI and pressure drop in idealized models of various types of vessel obstruction. Finally, the relations between DVI, FFR, hyperemic flow velocity, and anatomical lesion factors were investigated in patients with intermediate coronary stenoses, who had participated in a prospective, international, multicenter study with high-quality coronary angiographic evaluation and pressure wire-based FFR measurement(16). 


\section{Methods}

Virtual models of obstructed vessel. For the computer-based analysis of pulsatile flow in coronary stenoses, 3 groups of 9 idealized virtual vessel models (A1-3, B1-3, C1-3) were generated that represented obstructions with different characteristics (Figure 2). All models had a reference lumen diameter of $3.0 \mathrm{~mm}$ and an obstruction in the middle, with normal segments of $10 \mathrm{~mm}$ length proximal and distal to the obstruction. There were three degrees of diameter stenosis (DS\%) in each group: 33\%, 50\%, and 67\%. For models of the same group, other geometric lumen characteristics were the same. The lesion length (LL) was $10 \mathrm{~mm}$ for models in Group A and C, and 20mm for models in Group B. Models in Group A and B had concentric obstructions with a smooth transition between obstructed and normal segments, while models in Group C had eccentric obstructions with a steep transition.

Study population. To investigate in vivo the relation between local flow patterns, DVI and FFR, we performed a post-hoc analysis of data of the FAVOR (Functional Assessment by Various Flow Reconstructions) Pilot Study, a prospective multicenter study to investigate the accuracy of angiography-based computational FFR versus conventional pressure wire-based FFR as the reference(16). The institutional review boards of the individual study centers and, as appropriate, the local and/or national medical ethical committees approved the protocol of the FAVOR pilot study; all patients provided a written informed consent. The study population consisted of 73 patients ( 84 vessels) in the final analysis; characteristics of the patients population and the assessed coronary lesions, and the primary end-point have been reported(16). Mean age was $65.8 \pm 8.9$ years and 61 (83.5\%) patients were male(16). FFR was measured in left main stem $(n=1)$, left anterior descending artery $(n=46)$, diagonal branch $(n=1)$, left circumflex artery $(n=12)$, obtuse marginal branch $(n=5)$, and right coronary artery $(n=19)$. Key characteristics of the interrogated vessels are as follows: minimum lumen

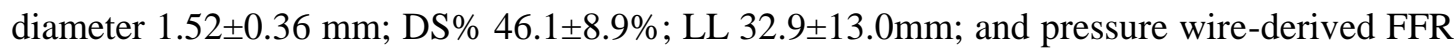
$0.84 \pm 0.08$ (median 0.85 [IQR 0.74-0.89])(16).

Three-dimensional (3D) angiographic reconstruction. The 3D geometry of the coronary tree was reconstructed by an experienced analyst who used a well-validated, established method(17,18). In summary, two angiographic image runs with minimal overlap and 
foreshortening were selected. The interrogated main vessel and its side branches of $>1 \mathrm{~mm}$ diameter were separately reconstructed by a validated 3D quantitative coronary angiography software (QAngio XA 3D Research Edition 2.2.3, Medis Special bv, Leiden, the Netherlands). Subsequently, the main vessel and its side branches were merged into a coronary tree structure using an in-house developed software package (Q3DMerge). Minimum lumen diameter, minimum lumen area, reference vessel diameter, DS\%, percent area stenosis, and bifurcation angle (if applicable) were quantified. For interrogated vessels with serial lesions, the lengths of all lesions were added up.

Mean volumetric flow rate. For each virtual vessel model, we performed a total of 10 simulations, assuming different mean volumetric flow rates at the inlet of the model (range: $42.4 \mathrm{~mL} / \mathrm{min}-424.0 \mathrm{~mL} / \mathrm{min}$ ). For the in-vivo analysis, TIMI frame count analysis was performed on angiographic image runs that were acquired before and during pharmacologically induced hyperemia. Patient-specific mean volumetric flow rates at baseline and hyperemia were obtained by combining the dye flow time and coronary tree volume by the $3 \mathrm{D}$ reconstruction(17).

Computational fluid dynamics (CFD). Geometries of virtual vessel models and the reconstructed coronary trees were discretized into tetrahedral meshes using ANSYS ICEM software (vers. 15.0, ANSYS, Inc., Canonsburg, PA). The amount of tetrahedral elements was approximately 90,000 for virtual models and 200,000 to 1 million for reconstructed coronary trees. Navier-Stokes equations were implemented in each mesh and solved using the finite volume method by ANSYS Fluent software (vers. 15.0, ANSYS, Inc.). Blood was assumed to be a Newtonian fluid with a viscosity of $0.00345 \mathrm{~kg} / \mathrm{m} \bullet \mathrm{s}$ and a density of $1060 \mathrm{~kg} / \mathrm{m}^{3}$. Non-slip boundary condition was applied on the artery wall. A pulsatile flow waveform(19) with the average velocity value matching with the aforementioned mean hyperemic flow rate was imposed at the vessel inlet. At the outlets of the interrogated vessel and its side branches, fully developed flow condition was applied. Flow separation at the bifurcations of the reconstructed coronary tree was determined by the bifurcation angles and the reference diameters of the two daughter branches(17). The computations were considered converged when the maximum change in hemodynamic parameters between iterations was $<0.1 \%$. 
Computation of DVI. After CFD simulation, the flow velocity and its direction at each mesh was obtained, from which the flow vorticity was automatically calculated by ANSYS CFD-POST software (version 15.0, ANSYS, Inc.) according to the following formula(12):

$$
\begin{aligned}
\text { vorticity } & =\nabla \times V=(\partial / \partial x, \partial / \partial y, \partial / \partial z) \times\left(V_{x}, V_{y}, V_{z}\right) \\
& =\left(\partial V_{z} / \partial y-\partial V_{y} / \partial z, \partial V_{x} / \partial z-\partial V_{z} / \partial x, \partial V_{y} / \partial x-\partial V_{x} / \partial y\right)
\end{aligned}
$$

$\partial / \partial x \partial / \partial y$, and $\partial / \partial z$ denote the derivative operators and $V_{x}, V_{y}, V_{z}$ are the components of the fluid velocity vector $V$ in $x, y$, and $z$ directions. In mathematics, vorticity is the curl or rotational of the velocity field $V(12)$. It contains information about two motions: shear and rotation and therefore the magnitude of vorticity is determined by both the flow velocity and its direction. Disturbed vorticity index (DVI) was defined as follows:

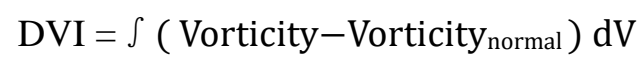

where Vorticity $y_{\text {normal }}$ represents normal flow vorticity in the hypothetical absence of the stenosis, which can be calculated based on the reference vessel surface obtained from 3D angiographic reconstructions and quantitative coronary angiographic data (20). In this study, DVI was calculated by integrating the difference between the actual vorticity and the normal vorticity in the entire vessel in order to account for the entire flow disturbance along the interrogated vessel until the position where the FFR was measured.

Statistical analysis. Normal distribution was tested with the Shapiro-Wilk test. Continuous variables are expressed as mean $\pm \mathrm{SD}$ or as median (interquartile range [IQR]), as appropriate, and categorical variables are expressed as frequencies (\%). Correlation analysis and regression modeling were implemented to investigate the relationships among continuous variables, denoted by Spearman's rank correlation coefficient (rho) $\rho$ and determination coefficient $R^{2}$, respectively. The interrogated in-vivo vessels were divided into two sub-groups with equal numbers of vessels, according to the median of LL and hyperemic flow velocity, respectively. Mann-Whitney's test or independent t-test was used to compare the differences between sub-groups. P-values were two-sided and considered significant if $<0.05$. Data were analyzed on a per-patient basis for clinical characteristics and on a per-vessel basis for all other calculations. All statistical analyses were performed with SPSS version 20.0 (SPSS Inc., Chicago, IL). 


\section{Results}

\section{Computerized Assessment of Virtual Vessel Models}

\section{DVI, lesion characteristics, and flow rate.}

Factors that disturb laminar flow affected the value of DVI. As shown in Figure 3, DVI increased with increasing inlet flow rate in all models. Considering the effect of the geometric characteristics of the models, DVI increased with lesion severity and complexity. For models in the same group, a greater degree of DS\% led to higher DVI. Models of long obstructions (Group B) and eccentric obstructions (Group C) had higher DVI than short concentric obstructions (Group A).

Relation between DVI and pressure drop across models. DVI was found to have an obvious curve correlation with pressure drop in all obstructed models (Figure 4). When applying a fully quadratic model, the coefficient of determination $\mathrm{R}^{2}$ was $0.85(\mathrm{p}<0.001)$. The models with severe stenoses (A3, B3, and C3) showed a greater pressure drop and a higher DVI with different growth slopes as compared to models with less severe stenoses.

\section{In-Vivo Assessment of Intermediate Coronary Lesions}

Computed hemodynamic parameters and DVI of obstructed vessels in-vivo. In 84 vessels with intermediate lesions from 73 patients, the median (IQR) values of computed hemodynamic parameters were: baseline volumetric flow rate $82.9 \mathrm{~mL} / \mathrm{min}(63.4-109.7)$ and hyperemic volumetric flow rate $166 \mathrm{~mL} / \mathrm{min}$ (130.8-193.7)). In addition, vorticity distribution and flow patterns were quantified in all 84 vessels. The median value (IQR) of DVI was $8.82 \mathrm{E}+04\left(\mathrm{~mm}^{3} / \mathrm{s}\right)(5.47 \mathrm{E}+04-1.34 \mathrm{E}+05)$. Heterogeneous flow vorticity was accompanied by disturbed flow patterns. Figure 5 shows an example of local flow patterns and vorticity distribution in an obstructed LAD (see also Appendix 1 for a velocity vector video). The stenosis accelerated flow velocity and generated highly disturbed flow patterns in the post-stenotic region (Figure 5A). The magnitude of vorticity increased rapidly in the upstream part of the stenosis, reaching the highest value at the narrowest lesion site (Figure 5B). Distal to that, the magnitude of vorticity decreased gradually, and its direction altered sharply in the post-stenotic region, which could be best observed in cross-section profiles (Figure 5C). 
Correlation between DVI, FFR, hyperemic flow velocity, and anatomical parameters. As shown in Figure 6, there was an inverse correlation between FFR and DVI $(\rho=-0.74, p<0.001)$ that was stronger than the relation of FFR with hyperemic flow velocity $(\rho=-0.27, p=0.015)$ and with anatomic parameters, including DS\% $(\rho=-0.40, p<0.001)$ and $\operatorname{LL}(\rho=-0.36, p=0.001)$. DVI showed a moderate correlation with LL $(\rho=0.44, p<0.001)$ and $\operatorname{DS} \%(\rho=0.23, p=0.037)$.

Sub-group analysis according to lesion length. The LL was $43.6 \pm 7.6 \mathrm{~mm}$ for the long-lesion group and $22.2 \pm 7.0 \mathrm{~mm}$ for the short-lesion group. Both DS\% and mean hyperemic flow velocity showed no significant difference between the two groups $(p=0.44$ and $\mathrm{p}=0.42$, respectively). However, the long-lesion group had on average a lower FFR $(0.80 \pm 0.09$ versus $0.87 \pm 0.06, \mathrm{p}<0.001)$ and a higher DVI $\left(1.3 \mathrm{E}+05 \mathrm{~mm}^{3} / \mathrm{s}[8.3 \mathrm{E}+04-\right.$ $1.6 \mathrm{E}+05]$ versus $\left.6.5 \mathrm{E}+04 \mathrm{~mm}^{3} / \mathrm{s}[4.4 \mathrm{E}+04-9.0 \mathrm{E}+04], \mathrm{p}<0.001\right)$ than the short-lesion group (Table 1).

Sub-group analysis according to hyperemic flow velocity. Mean hyperemic velocity was $0.46 \mathrm{~m} / \mathrm{s}$ for the high-velocity group and $0.29 \mathrm{~m} / \mathrm{s}$ for the slow-velocity group. There was no significant difference in DS\% or LL between the two groups $(\mathrm{p}=0.29$ and $\mathrm{p}=0.38$, respectively). However, the high-velocity group showed on average a higher DVI (1.0E+05 $\mathrm{mm}^{3} / \mathrm{s}[7.8 \mathrm{E}+04-1.5 \mathrm{E}+05]$ versus $\left.6.7 \mathrm{E}+04 \mathrm{~mm}^{3} / \mathrm{s}[3.6 \mathrm{E}+04-1.1 \mathrm{E}+05], \mathrm{p}=0.002\right)$ and a lower FFR $(0.82 \pm 0.07$ versus $0.86 \pm 0.09, \mathrm{p}=0.061)$ than the slow-velocity group (Table 1$)$. 


\section{Discussion}

We developed a novel hemodynamic parameter to quantitatively assess disturbed coronary flow. This parameter, named DVI, was related to both, geometric characteristics of vascular obstruction and coronary flow rate. In addition, DVI showed strong correlations with pressure drop across idealized virtual vessel models and with pressure wire-based FFR in coronary arteries with intermediate lesions in-vivo.

The aforementioned relations are plausible, as a reduction in FFR indicates more pressure drop during hyperemia, that may be closely related to energy loss, caused by a disturbance of coronary blood flow(21). However, prior to the current study, the relation between the extent of flow disturbance and FFR has never been demonstrated. As a consequence, the findings of the present study are unique, and they may help us better comprehend the mechanisms underlying FFR.

In this study, we observed that the distribution of flow vorticity was homogeneous for undisturbed laminar flow. Obstruction increased the magnitude of vorticity near the vessel wall in the narrowest position and lead to a heterogeneously separated distribution of vorticity with an increased magnitude near the vessel center in post-stenotic regions. DVI was a metric to quantify disturbed flow on the basis of the abnormal vorticity by subtracting the normal part, leaving only fluctuations of vorticity caused by the stenosis. In healthy segments, the remaining fluctuated vorticity is theoretical zero. Therefore, the DVI accumulated all fluctuated vorticity in the vessel in which the FFR was measured.

Lessons from assessing the idealized vessel models. The assessment of the idealized vessel models revealed that DVI increased with fluid flow rate, lesion severity, and lesion complexity. The higher DVI is, the more mechanical energy is consumed to drive flow through the obstruction models. The total mechanical energy of fluid equals the sum of kinetic energy plus potential energy. Since the mean velocity of fluid increases in narrowed segments but decreases in distal unobstructed segments to the initial level, kinetic energy does not change along entire obstructed vessel models. Consequently, the loss of mechanical energy, which predominantly can be attributed to irreversible friction loss that produces heat, results from a reduction in potential energy that manifests as a drop of distal pressure. In other 
words: a greater DVI indicates the presence of a greater loss of mechanical energy that is required to overcome friction in a stenosis, which finally results in more lowering of pressure distal to the obstruction. This explains the strong relation between DVI and pressure drop observed.

Although the growth trends between pressure drop across models and DVI were quite similar, different geometric features of obstructions resulted in slightly different growth slopes, especially for the most obstructed model in Group B and C (Figure 4). This might be explained by the different sources of mechanical energy consumption by transiting pulsatile flow(21). DVI increased in obstructed coronary vessels with severely disturbed flow, and a higher DVI means a greater likelihood of post-stenotic turbulence of flow. Because of the abrupt vessel obstruction and expansion for the most stenotic model in Group B and C, there was widespread laminar-turbulent transition in the post-stenotic region, which increases energy loss on top of the aforementioned irreversible friction loss. In the less severely obstructed models with DS\% of $33 \%$ and 50\%, mechanical energy consumption was predominately based on viscous resistance. Therefore, the most severely obstructed model -especially in complex obstructions (Group C)- caused more pressure drop than the less severely obstructed models.

DVI in-vivo and its relation with FFR and anatomy parameters. DVI is correlated with the anatomical lesion parameters LL and DS\%. Vessels in the long-lesion group and the high-velocity group had higher DVI than vessels in the short-lesion or low-velocity groups. The mechanisms by which flow rate and stenosis characteristics act upon DVI are quite similar to the effects of coronary flow and changes in lumen geometry upon FFR. This suggests a potential intrinsic relationship between DVI and FFR, as was finally demonstrated in-vivo in patients with intermediate coronary lesions. Due to fact that multiple factors influence the regulation of myocardial blood flow and lesions in diseased human coronary arteries are highly diverse, it is unrealistic to expect in vivo simple and intuitive fitting curves as seen for the correlations in the virtual vessel models. Nevertheless, in 84 coronary vessels with intermediate lumen obstruction, we found a strong inverse correlation between DVI and FFR $(\rho=-0.74, p<0.001)$ that was stronger than the correlation between hyperemic flow velocity and FFR $(\rho=-0.27, p=0.015)$. 
The correlations between FFR and anatomy parameters, on the other hand, showed more scatter and were only moderate ( $\rho=-0.40$ for DS\% and $\rho=-0.36$ for LL). This is not surprising, as DS\% and LL are only two of several anatomical parameters relevant to FFR. The lack of hemodynamic information explains why the sole use of the DS\% or LL is far from sufficient to reflect true coronary physiology. This is in line with previous studies that reported a limited usefulness of anatomical parameters to predict FFR(22-24).

Our results confirm that a reduction in FFR is closely related to energy loss along stenotic vessel and that disturbance of laminar flow(21) contributes significantly to FFR, besides other established factors such as coronary flow and anatomical lesion characteristics(11). The findings highlight the intrinsic relationship between FFR and local coronary flow patterns, and they partially explain the mechanics why FFR works clinically.

Implications for clinical research. Different flow patterns directly determine endothelial cell morphology, metabolism, and inflammatory phenotypes through signal transduction and gene expression(25). Researches have concentrated upon the velocity gradient near the endothelium, represented by wall shear stress, to investigate interactions between hemodynamics and the progression of atherosclerosis. Nevertheless, the role of different types of wall shear stress on the progression of atherosclerosis is still a matter of discussion. While low and oscillatory wall shear stress is generally considered the prevailing mechanism for the initiation and (early) progression of atherosclerosis $(6,26,27)$, this concept was recently questioned(28), as high wall shear stress has also been proposed to be a key regulator of vessel wall inflammation, development of high-risk plaques, and plaque rupture $(9,29)$. One of the reasons for this inconsistence might be the fact that the extent of flow disturbance has generally been ignored. Vorticity contains the information about fluid shear that is provided by wall shear stress, and it also describes the tendency of flow to rotate. Hence, vorticity might represent a more profound stimulus to endothelial cells.

In the context of the progression of atherosclerosis, the impact of disturbed flow and vorticity may not be fully appreciated. The findings of the present study suggest that additional prospective explorations are warranted of the role of vorticity for the pathological processes that lead to progression of atherosclerosis, a disease that culminates at certain spots of the coronary tree in the development of advanced atheromas. 
Furthermore, DVI reflects in-vivo the accumulated effect of all disturbed flows along the stenotic segments of a vessel, influenced by DS\%, LL, perfusion flow, and other geometrical and hemodynamic variables. The strong inverse relation between DVI and FFR in intermediate coronary lesions, which was superior to the relation of FFR with anatomical parameters and hyperemic coronary flow velocity, suggests that mechanistic studies with DVI assessment might help us to better understand the relation between lesion geometry, FFR data, and clinical prognosis(30). Studies to assess approaches using DVI for a potential refinement of image-based (i.e. non-pressure wire-based) FFR computation are of great interest.

Study limitations. In the present study, the idealized virtual models of obstructed arteries assumed the vessels to be rigid cylindrical tubes. Nevertheless, atherosclerosis generally reduces a vessel's elastic properties as compared to when it was healthy; as a result, vessels may transform into rigid tubes that are particularly stiff in the presence of concentric atherosclerotic disease and significant calcification. In addition, most vessels are not entirely straight, and lumen shape may not be round in many diseased segments. Nevertheless, while biology is certainly more complex than our virtual models, the assessment of the models helped us better comprehend the relations between DVI, flow rate, geometric characteristics of obstructions, and pressure drop. Theoretically, normal vorticity should in-vivo be calculated from a reference vessel (assuming no lesions), taking into account the step-down phenomenon of all bifurcations; however, this would greatly increase the amount of calculation required for diseased coronary arteries. Nevertheless, all coronary vessels, included in the present study, had relatively normal segments at their proximal and distal ends, from where vorticity was taken as a reasonable substitute for truly normal vorticity.

\section{Conclusions}

DVI, a novel index to quantify disturbed flow was correlated with flow velocity and with the severity and complexity of lumen obstruction. DVI was related to pressure drop in different obstructed virtual models and showed a strong inverse relation with FFR in intermediate human coronary lesions in vivo. Assessment of DVI may improve our understanding of the relations between lesion geometry, hemodynamics, and FFR. Additional research on DVI is 
warranted, as it may help to better understand FFR and to evaluate whether the use of DVI could further improve the image-based computation of FFR.

\section{Acknowledgements:}

$\mathrm{S} \mathrm{Tu}$ acknowledges the support by The Program for Professor of Special Appointment (Eastern Scholar) at Shanghai Institutions of Higher Learning (No. TP2015014). 


\section{CLINICAL PERSPECTIVES}

\section{What is known?}

FFR is the cornerstone of physiological assessment of coronary lesions and local flow patterns play an essential role in the formation and progression of atherosclerosis.

\section{What is new?}

A novel hemodynamic parameter, Disturbed Vorticity Index (DVI), was developed for quantitative assessment of disturbed coronary flow, which correlates well with FFR in vivo, indicating a strong association between FFR and local flow disturbance.

\section{What is next?}

Use of DVI might further improve the accuracy of image-based computation of FFR. Further assessment of the role of vorticity for the pathological processes that lead to progression of atherosclerosis is of interest. 


\section{References}

1. Tonino PA, De Bruyne B, Pijls NH et al. Fractional flow reserve versus angiography for guiding percutaneous coronary intervention. N Engl J Med 2009;360:213-24.

2. De Bruyne B, Pijls NH, Kalesan B et al. Fractional flow reserve-guided $\mathrm{PCl}$ versus medical therapy in stable coronary disease. N Engl J Med 2012;367:991-1001.

3. Fearon WF, Shilane D, Pijls NH et al. Cost-effectiveness of percutaneous coronary intervention in patients with stable coronary artery disease and abnormal fractional flow reserve. Circulation 2013;128:1335-40.

4. Pijls NH, van Schaardenburgh P, Manoharan G et al. Percutaneous coronary intervention of functionally nonsignificant stenosis: 5-year follow-up of the DEFER Study. J Am Coll Cardiol. 2007;49:2105-11.

5. Wentzel JJ, Chatzizisis YS, Gijsen FJ, Giannoglou GD, Feldman CL, Stone PH. Endothelial shear stress in the evolution of coronary atherosclerotic plaque and vascular remodelling: current understanding and remaining questions. Cardiovasc Res 2012;96:234-43.

6. Stone PH, Saito S, Takahashi S et al. Prediction of progression of coronary artery disease and clinical outcomes using vascular profiling of endothelial shear stress and arterial plaque characteristics: the PREDICTION Study. Circulation 2012;126:172-81.

7. Thondapu V, Bourantas CV, Foin N, Jang IK, Serruys PW, Barlis P. Biomechanical stress in coronary atherosclerosis: emerging insights from computational modelling. Eur Heart J. in press; published online ahead of print on February 22, 2016 (DOI: 10.1093/eurheartj/ehv689).

8. Zarins CK, Giddens DP, Bharadvaj BK, Sottiurai VS, Mabon RF, Glagov S. Carotid bifurcation atherosclerosis. Quantitative correlation of plaque localization with flow velocity profiles and wall shear stress. Circ Res 1983;53:502-14.

9. Samady H, Eshtehardi P, Mcdaniel MC et al. Coronary artery wall shear stress is associated with progression and transformation of atherosclerotic plaque and arterial remodeling in patients with coronary artery disease. Circulation 2011;124:779-88.

10. Fry DL. Acute vascular endothelial changes associated with increased blood velocity gradients. Circ Res 1968;22:165-97.

11. Johnson NP, Kirkeeide RL, Gould KL. Coronary anatomy to predict physiology fundamental limits. Circ Cardiovasc Imaging 2013;6:817-832.

12. Wu JZ, Ma HY, Zhou MD. Vorticity Kinematics. Vorticity and Vortex Dynamics. Berlin, Heidelberg: Springer Berlin Heidelberg, 2006:67-129.

13. Bernad ES, Bernad SI, Craina ML. Hemodynamic parameters measurements to assess severity of serial lesions in patient specific right coronary artery. Biomed Mater Eng 2014;24:323-34.

14. Ishikawa T, Oshima S, Yamane R. Vortex enhancement in blood flow through stenosed and locally expanded tubes. Fluid Dyn Res 2000;26:35-52.

15. Ramaswamy SD, Vigmostad SC, Wahle A et al. Fluid Dynamic Analysis in a Human Left Anterior Descending Coronary Artery with Arterial Motion. Ann Biomed Eng 2004;32:1628-1641.

16. Tu S, Westra J, Yang J et al. Diagnostic Accuracy of Fast Computational Approaches to Derive Fractional Flow Reserve From Diagnostic Coronary Angiography : The International Multicenter FAVOR Pilot Study. J Am Coll Cardiol Intv 2016;9:2024-2035. 
17. Tu S, Barbato E, Köszegi Z et al. Fractional Flow Reserve Calculation From 3-Dimensional Quantitative Coronary Angiographyand TIMI Frame Count : A Fast Computer Model to Quantify the Functional Significance of Moderately Obstructed Coronary Arteries. J Am Coll Cardiol Intv 2014;7:768-777.

18. Liu L, Yang W, Nagahara Y et al. The impact of image resolution on computation of fractional flow reserve: coronary computed tomography angiography versus 3-dimensional quantitative coronary angiography. Int J Cardiovasc Imaging 2016;32:513-523.

19. Barlis P, Poon EK, Thondapu $V$ et al. Reversal of flow between serial bifurcation lesions: insights from computational fluid dynamic analysis in a population-based phantom model. Eurolntervention 2015;11:e1-3.

20. Tu S, Holm NR, Koning G, Reiber JHC. The impact of acquisition angle differences on three dimensional quantitative coronary angiography. Catherter Cardio Inte 2011;78:214 - 222.

21. Flanigan D, Tullis JP, Streeter V, Whitehouse Jr W, Fry WJ, Stanley JC. Multiple subcritical arterial stenoses: effect on poststenotic pressure and flow. Ann Surg 1977;186:663.

22. Toth $G$, Hamilos $M$, Pyxaras $S$ et al. Evolving concepts of angiogram: fractional flow reserve discordances in 4000 coronary stenoses. Eur Heart J 2014;35:2831-8.

23. Tu S, Echavarria-Pinto $M$, von Birgelen $C$ et al. Fractional Flow Reserve and Coronary Bifurcation Anatomy : A Novel Quantitative Model to Assess and Report the StenosisSeverity of Bifurcation Lesions. J Am Coll Cardiol Intv;8:564-574.

24. Park SJ, Kang SJ, Ahn JM et al. Visual-functional mismatch between coronary angiography and fractional flow reserve. J Am Coll Cardiol Intv 2012;5:1029-1036.

25. Pan S. Molecular mechanisms responsible for the atheroprotective effects of laminar shear stress. Antioxid Redox Sign 2009;11:1669-82.

26. Ku DN, Giddens DP, Zarins CK, Glagov S. Pulsatile flow and atherosclerosis in the human carotid bifurcation. Positive correlation between plaque location and low oscillating shear stress. Arterioscler Thromb Vasc Biol 1985;5:293-302.

27. Cecchi E, Giglioli C, Valente $S$ et al. Role of hemodynamic shear stress in cardiovascular disease. Atherosclerosis 2011;214:249-256.

28. Peiffer V, Sherwin SJ, Weinberg PD. Does low and oscillatory wall shear stress correlate spatially with early atherosclerosis? A systematic review. Cardiovasc Res 2013;99:242-250.

29. Fukumoto Y, Hiro T, Fujii T et al. Localized elevation of shear stress is related to coronary plaque rupture: a 3-dimensional intravascular ultrasound study with in-vivo color mapping of shear stress distribution. J Am Coll Cardiol 2008;51:645-650.

30. Johnson NP, Tóth GG, Lai D et al. Prognostic value of fractional flow reserve: linking physiologic severity to clinical outcomes. J Am Coll Cardiol 2014;64:1641-1654. 


\section{Figure Legends}

\section{Figure 1. Schematic illustration of velocity and vorticity in a stenotic vessel.}

Stenosis accelerates flow velocity and generates reversed flow in the post-stenotic region. The vorticity magnitude of laminar flow in normal segment increases along the radial direction, with zero value in the vessel center and maximum on the wall, where shear is the largest. The vorticity direction is perpendicular to the vessel centerline and it obeys the 'right-hand rule': when the fingers of the right hand are curled in the direction of vorticity, the right thumb points in the direction of antegrade flow.

Figure 2. Setup for virtual assessment of pulsatile flow in computer models of idealized artery.

The length of models was $30 \mathrm{~mm}, 40 \mathrm{~mm}$, and $30 \mathrm{~mm}$ for Group A, B, and C, respectively. The minimal lumen diameter of the three degrees of obstruction was $2 \mathrm{~mm}(\mathrm{DS} \%=33 \%), 1.5$ $\mathrm{mm}(\mathrm{DS} \%=50 \%)$, and $1 \mathrm{~mm}(\mathrm{DS} \%=67 \%)$, respectively. Models in Group A and B had concentric obstructions with a smooth transition, while models in Group C had eccentric obstructions with a steep transition.

DS\%=percent diameter stenosis.

\section{Figure 3. Factors affect DVI value in different virtual vessel models.}

The disturbed vorticity index (DVI) increased with inlet flow rate for all models. Within same groups, models with higher degree of obstruction produced higher DVI under the condition of the same inlet flow rate. Long obstructions and eccentric obstructions with a steep transition increased DVI.

DVI=disturbed vorticity index

Figure 4. Correlation between DVI and pressure drop in different virtual vessel models.

There was a curve correlation between DVI and pressure drop across all models. The growth trends of different models were quite similar but influenced by geometric features of the obstructions. 
DVI=disturbed vorticity index

Figure 5. Local flow patterns in an obstructed left anterior descending (LAD) artery.

(A) Velocity vector shows a highly disturbed flow and a recirculation zone in the post-stenotic region (arrow). (B) Vorticity vector in the vicinity of the stenosis. (C) Vorticity magnitude counter (first row) and distribution profile (second row) in the cross-section views. In the normal cross section (plane 1), distribution of flow vorticity was homogeneous. In the post-stenotic segment (plane 3 and plane 4), heterogeneous flow vorticity was observed. According to the 'right-hand rule', clockwise low magnitude vorticity in the upper right of plane 3 and heterogeneously separated vorticity in the opposite direction of plane 4 co-localized in the region of flow recirculation.

$\mathrm{LAD}=\mathrm{left}$ anterior descending coronary artery.

Figure 6. Assessment of relations between DVI, FFR, DS\%, and LL in patients with intermediate coronary lesions

There was a good inverse correlation between DVI and FFR (Spearman's $\rho=-0.74, p<0.001$ ) (A), which was better than the correlation between hyperemic flow velocity and FFR $(\rho=-0.27$, $\mathrm{p}=0.015)(\mathbf{B})$. FFR and DVI showed a moderate correlation with anatomic parameters, including LL $[\rho=-0.36, p=0.001$ for FFR $(\mathbf{C})$ and $\rho=0.44, p<0.001$ for DVI (D)] and DS\% $[\rho=-0.40, p<0.001$ for FFR $(\mathbf{E})$ and $\rho=0.23, p=0.037$ for DVI $(\mathbf{F})]$.

DVI=disturbed vorticity index; FFR= fractional flow reserve; DS\%= percent diameter stenosis; LL= lesion length.

\section{Video Legends}

Video 1 (Appendix). Cyclic change of flow velocity of an obstructed left anterior descending artery. 


\section{Tables}

Table 1. Differences between FFR, DVI, and DS\% between sub-groups, based on lesion length and hyperemic flow velocity

\begin{tabular}{|c|c|c|c|c|c|c|}
\hline & \multicolumn{3}{|c|}{ Lesion length } & \multicolumn{3}{|c|}{ Hyperemic flow velocity } \\
\hline & $\begin{array}{l}\text { Long } \\
\text { lesions }\end{array}$ & $\begin{array}{l}\text { Short } \\
\text { lesions }\end{array}$ & $\Delta$, P-value & $\begin{array}{l}\text { High } \\
\text { velocity }\end{array}$ & $\begin{array}{l}\text { Low } \\
\text { velocity }\end{array}$ & $\Delta$, P-value \\
\hline FFR & $0.80 \pm 0.09$ & $0.87 \pm 0.06$ & $0.07, p<0.001$ & $0.82 \pm 0.07$ & $0.86 \pm 0.09$ & $0.03, p=0.061$ \\
\hline & $1.3 \mathrm{E}+05$ & $6.5 E+04$ & & $1.0 \mathrm{E}+05$ & $6.7 E+04$ & \\
\hline DVI & {$[8.3 \mathrm{E}+04$} & {$[4.4 \mathrm{E}+04$} & $5.2 E+04$ & {$[7.8 \mathrm{E}+04$} & {$[3.6 \mathrm{E}+04$} & $3.5 E+04$ \\
\hline$\left(\mathrm{mm}^{3} / \mathrm{s}\right)$ & $-1.6 \mathrm{E}+05]$ & $-9.0 E+04]$ & $p<0.001$ & $-1.5 E+05]$ & $-1.1 \mathrm{E}+05]$ & $p=0.002$ \\
\hline DS\% & $46.9 \pm 8.9$ & $45.4 \pm 8.9$ & $1.507, p=0.44$ & $45.1 \pm 9.0$ & $47.2 \pm 8.8$ & $2.1, p=0.29$ \\
\hline
\end{tabular}

Values are $\mathrm{n}(\%)$, mean $\pm \mathrm{SD}$ or median $[\mathrm{IQR}] . \Delta=$ difference; $\mathrm{DS} \%=$ percent diameter stenosis; DVI = disturbed vorticity index; FFR = fractional flow reserve; $\mathrm{IQR}=$ interquartile range. 
Figure 1

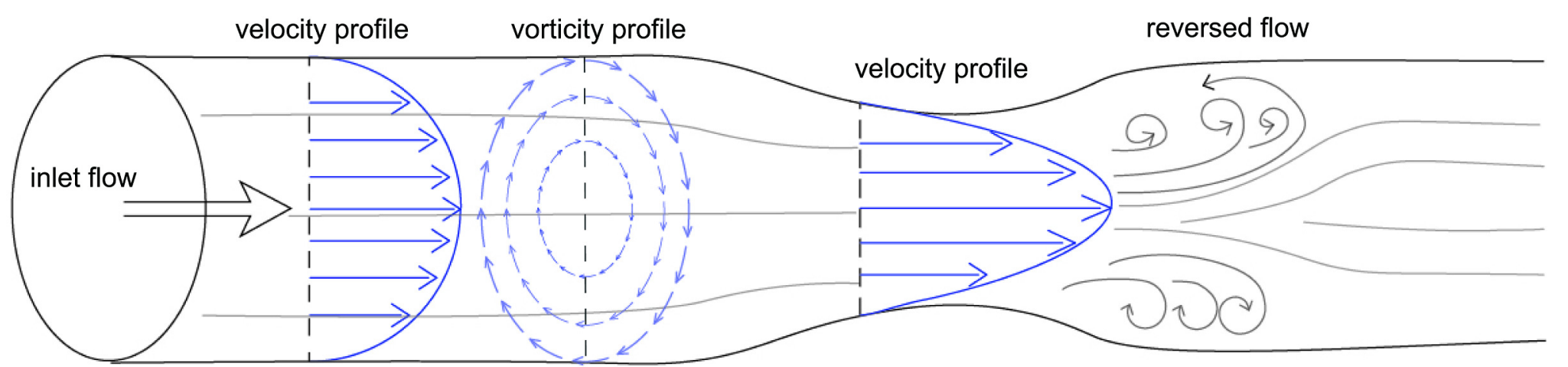


Figure 2

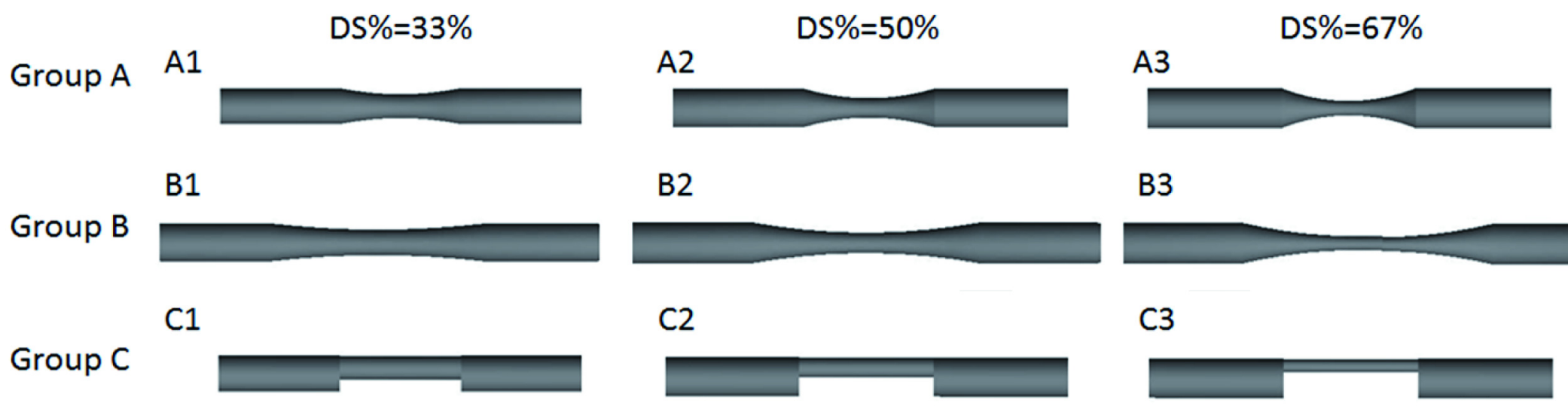


Figure 3






\section{Figure 5}

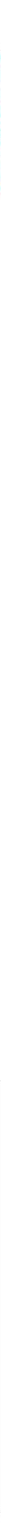


Figure 6

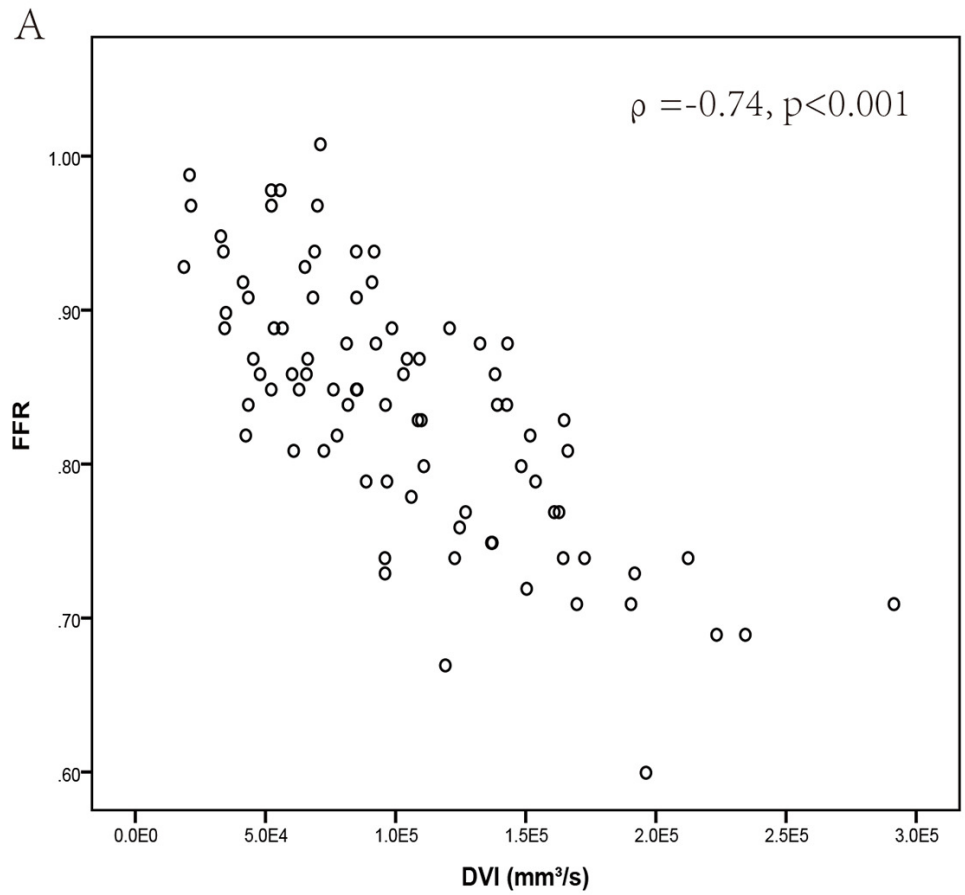

C
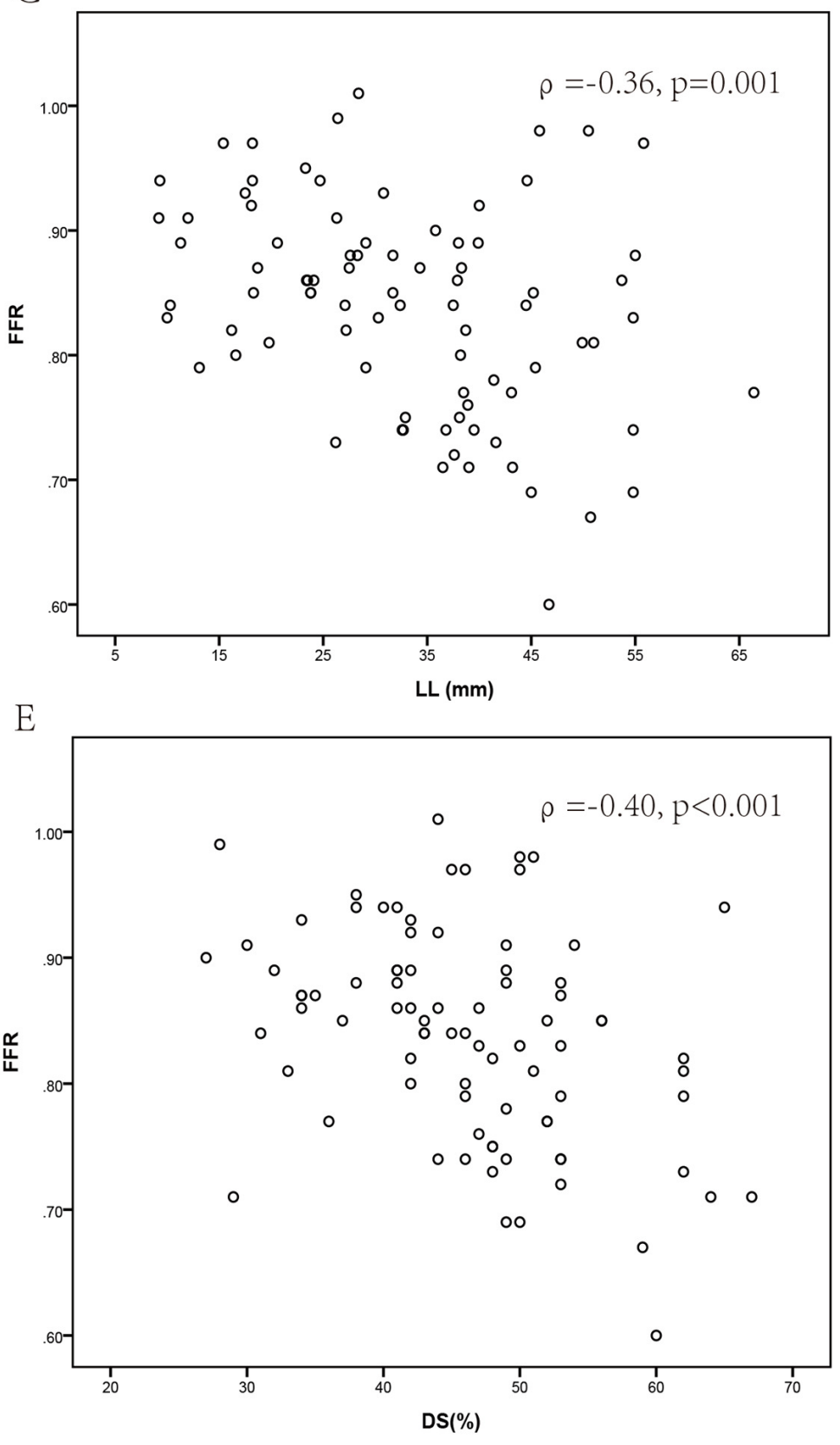
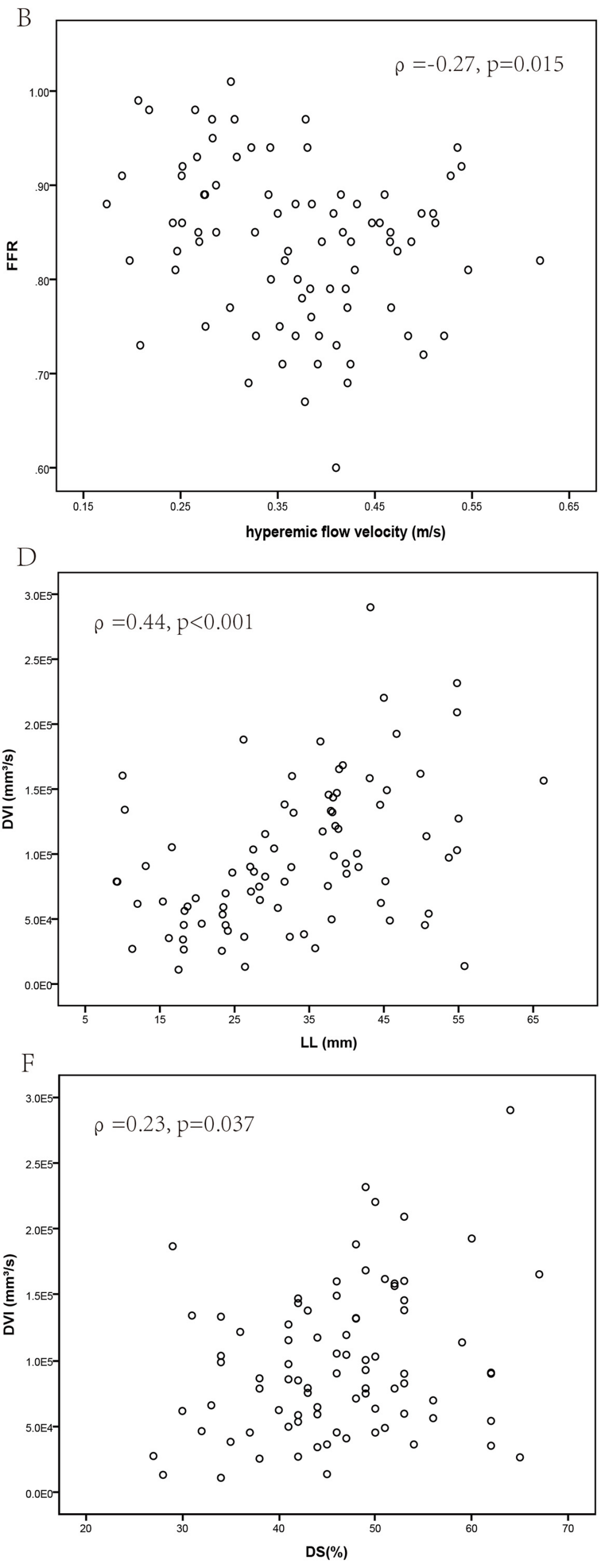